\title{
Efeitos e Aplicações do Campo Eletromagnético de Alta Intensidade (PEMF) em saúde e estética: perspectivas e evidências clínicas
}

\author{
Effects and Applications of the High Intensity Electromagnetic Field (PEMF) in health and \\ aesthetics: perspectives and clinical evidence \\ Efectos y Aplicaciones del Campo Electromagnético de Alta Intensidad (PEMF) en salud y estética: \\ perspectivas y evidencia clínica
}

Recebido: 10/13/2021 | Revisado: 10/19/2021 | Aceito: 10/20/2021| Publicado: 10/23/2021

Rodrigo Alvaro Brandão Lopes-Martins ORCID: https://orcid.org/0000-0003-4942-988X Universidade Brasil, Brazil E-mail: ralopesmartins@gmail.com

Michele Matias

ORCID: https://orcid.org/0000-0001-7467-329X Instituto de Pesquisas e Desenvolvimento Adoxy, Brazil E-mail: michele.matias@adoxy.com.br

Felicia Cadenas de Paiva Bueno

ORCID: https://orcid.org/0000-0003-1283-7628 Instituto de Pesquisas e Desenvolvimento Adoxy, Brazil E-mail: feliciabueno44@gmail.com Mayara Oguri

ORCID: https://orcid.org/0000-0001-7473-3125 Instituto de Pesquisas e Desenvolvimento Adoxy, Brazil E-mail: Mah_oguri@yahoo.com.br Michele Matias

ORCID: https://orcid.org/0000-0003-1283-7628 Instituto de Pesquisas e Desenvolvimento Adoxy, Brazil E-mail: michele.matias@adoxy.com.br Patrícia Sardinha Leonardo ORCID: https://orcid.org/0000-0002-7662-7702 Faculdade de Ciências da Saúde, Brazil E-mail: patyssardinha@gmail.com Carlos Ruiz-Silva

ORCID: https://orcid.org/0000-0002-6331-6032 Instituto de Pesquisas e Desenvolvimento Adoxy, Brazil E-mail: fisiocarlosruiz@gmail.com

\begin{abstract}
The increased interest and concern with physical fitness, not only from an athletic point of view, but also from an aesthetic point of view, has driven the search for new methods and technologies capable of helping to gain mass and muscle tone. Recent studies show that $81 \%$ percent of respondents reported dissatisfaction with their body image, even with 56\% having a normal body mass index. Pulsed electromagnetic field (PEMF) muscle stimulation technology uses alternating magnetic fields based on the law of electromagnetic induction to promote supramaximal muscle contractions. PEMF generates impulses that are independent of brain function, and with such a fast frequency that it does not allow the muscle relaxation phase, characterizing tetanic contractions. Electric currents and electromagnetism have been used in physical therapy and rehabilitation, especially for muscle strengthening. However, the PEMF technology has emerged as a more efficient and comfortable alternative for the patient, with the primary objective of toning and strengthening muscle groups. In this work, we performed a literature review of all scientific articles available and indexed in Pubmed and Web of Science about this technology in the last 20 years and its effects on skeletal muscles. We discuss the scientific evidence available from clinical studies and discuss effects and possible mechanisms of action on muscle contraction.
\end{abstract}

Keywords: Electromagnetic fields; PEMF; Skeletal muscle; Muscle contraction; Hypertrophy; Urinary incontinence.

\section{Resumo}

O aumento do interesse e da preocupação com a forma física, não somente do pronto de vista atlético, mas também estético vem impulsionando a busca por novos métodos e tecnologias capazes de auxiliar no ganho de massa e tônus 
muscular. Estudos recentes demonstram que $81 \%$ por cento dos entrevistados relataram insatisfação com sua imagem corporal, mesmo com $56 \%$ apresentando índice de massa corporal normal. A tecnologia de estimulação muscular por campo eletromagnético pulsado (PEMF) utiliza campos magnéticos alternados, baseado na lei da indução eletromagnética para promover contrações musculares supra máximas. O PEMF gera impulsos que são independentes da função cerebral, e com uma frequência tão rápida que não permite a fase de relaxamento muscular, caracterizando as contrações tetânicas. As correntes elétricas e o eletromagnetismo têm sido utilizados em fisioterapia e reabilitação, especialmente para o fortalecimento muscular. No entanto, a tecnologia de PEMF tem surgido como uma alternativa mais eficiente e menos incômoda para o paciente, com objetivo primordial de tonificação e fortalecimento de grupamentos musculares. Neste trabalho, realizamos uma revisão de literatura de todos os artigos científicos disponíveis e indexados no Pubmed e Web of Science nos últimos 20 anos sobre esta tecnologia e seus efeitos sobre a musculatura esquelética. Discorremos sobre as evidências científicas disponíveis a partir de estudos clínicos e discutimos efeitos e possíveis mecanismos de ação na contração muscular.

Palavras-chave: Campos eletromagnéticos; PEMF; Músculo esquelético; Contração muscular; Hipertrofia; Incontinência urinária.

\section{Resumen}

El creciente interés y preocupación por la forma física, no solo desde el punto de vista deportivo, sino también desde el punto de vista estético, ha impulsado la búsqueda de nuevos métodos y tecnologías capaces de ayudar a ganar masa y tono muscular. Estudios recientes muestran que el $81 \%$ de los encuestados informaron estar insatisfechos con su imagen corporal, incluso con un 56\% con un índice de masa corporal normal. La tecnología de estimulación muscular de campo electromagnético pulsado (PEMF) utiliza campos magnéticos alternos basados en la ley de inducción electromagnética para promover las contracciones musculares supramáximas. El PEMF genera impulsos independientes de la función cerebral, y con una frecuencia tan rápida que no permite la fase de relajación muscular, caracterizando las contracciones tetánicas. Las corrientes eléctricas y el electromagnetismo se han utilizado en fisioterapia y rehabilitación, especialmente para el fortalecimiento muscular. Sin embargo, la tecnología PEMF ha surgido como una alternativa más eficiente y menos incómoda para el paciente, con el objetivo principal de tonificar y fortalecer grupos musculares. En este trabajo, realizamos una revisión de la literatura de todos los artículos científicos disponibles e indexados en Pubmed y Web of Science sobre esta tecnología y sus efectos sobre la musculatura esquelética. Discutimos la evidencia científica disponible de los estudios clínicos y discutimos los efectos y posibles mecanismos de acción sobre la contracción muscular.

Palabras clave: Campos electromagnéticos; PEMF; Músculo esquelético; Contracción muscular; Hipertrofia; Incontinencia urinaria.

\section{Introdução}

O aumento do interesse e da preocupação com a forma física, não somente do pronto de vista atlético, mas também estético, impulsionado pela popularidade das redes sociais com a exposição do corpo, tem sido um fator importante na busca de tratamentos e novas tecnologias capazes de auxiliar na aquisição do "corpo perfeito". Procedimentos para o modelamento do contorno corporal tem apresentado uma procura crescente, incluindo lipossucção, criolipólise, correntes elétricas e mais recentemente, o campo eletromagnético pulsado. As sociedades de Cirurgia Plástica e estética, assim como a Associação de Cirurgiões Plásticos dos Estados Unidos da América reportaram mais de 250.000 procedimentos estéticos modeladores em 2018 (American Society of Plastic Surgeons, 2020; The American Society of Aesthetic Plastic Surgeons, 2018;).

Em 2019, um estudo realizado com mais de 300 estudantes da Arábia Saudita avaliou a satisfação dos jovens com o corpo, e sua relação com o índice de massa corporal e a imagem corporal. Os pesquisadores relataram que 81 por cento dos entrevistados relataram insatisfação com sua imagem corporal, e destes, 56\% apresentavam IMC normal (Radwan et al, 2019).

O contorno corporal é influenciado por três fatores principais, a saber: 1) a quantidade de tecido adiposo subcutâneo; 2) flacidez cutânea; e 3) a massa e o tônus muscular. Não obstante a variedade de tecnologias existentes para tratamentos de gordura localizada e flacidez dérmica, a questão do tônus muscular continua limitada ao exercício físico e correntes elétricas (Giesse, 2021; Alizadeh et al, 2016;). No entanto, o sistema muscular também colabora com o contorno corporal, possibilitando uma definição harmônica e estética através da tonificação muscular seja através de atividade física (Gentil, 2017) ou por estimulação de campo eletromagnético focado de alta intensidade (Kinney \& Lozanova, 2019; Kent \& Kinney, 2021; Halaas \& Bernardy, 2020). Esse tipo de tecnologia tem emergido como uma alternativa para a área de estética para a 
tonificação muscular.

Recentemente, Elena et al (2020) demonstraram, em um estudo controlado com 95 pacientes, o aumento significativo de tônus muscular na musculatura pélvica, e que esse aumento foi mais efetivo do que aquele observado com estimulação elétrica. Neste estudo, a tonificação muscular foi avaliada através de análise eletromiográfica da musculatura do assoalho pélvico, considerado o mais apropriado para tal objetivo.

As correntes elétricas e o eletromagnetismo têm sido utilizados em fisioterapia e reabilitação, especialmente para o fortalecimento muscular. No entanto, ao contrário das correntes elétricas, os campos eletromagnéticos não apresentam os problemas de dor e queimaduras como as correntes elétricas, sendo significativamente mais confortável para o paciente (McRae \& Boris, 2013).

O presente estudo visa realizar um levantamento bibliográfico nas principais bases de dados científicos, adotando uma metodologia de busca sistemática, sobre artigos científicos de estudos clínicos primários sobre a tecnologia de campo eletromagnético pulsado de alta intensidade e seus efeitos sobre o sistema muscular esquelético.

\section{Metodologia}

Como não há número suficiente de ensaios clínicos randomizados para a tecnologia de PEMF em Sistema Muscular Esquelético, decidimos realizar uma revisão integrativa para pesquisar a literatura de forma mais ampla. Apesar da falta de especificidade da revisão integrativa, uma vez que permite diversas metodologias (estudos teóricos e empíricos; Whittemore e Knafl, 2005), é conhecida por definir conceitos, revisar teorias e evidências, analisar a qualidade metodológica dos estudos e, por fim resumir os achados por meio de uma abordagem sistemática (Whittemore \& Knafl, 2005; Hopia et al., 2016).

\section{Estratégia de Busca}

Cinco bases de dados como Medline, Central, Scopus, Lilacs e PEDro foram pesquisadas para obter os estudos de 2001 a Setembro de 2021, este último mês foi o único que a pesquisa de banco de dados foi realizada.

Palavras-chave e termos MeSH foram usados e combinados por operadores booleanos da seguinte forma: (PEMF OU HIFEM AND Skeletal muscle ou muscle; Pulsed Electromagnetic Field AND Skeletal muscle ou muscle; Foram selecionados apenas estudo clínicos realizados em humanos, onde foi relatada aleatorização dos sujeitos participantes. Os resultados foram analisados pela equipe de pesquisadores e profissionais clínicos para elaboração da revisão teórica sobre os efeitos da estimulação de campo eletromagnético sobre a musculatura esquelética.

\section{O PEMF}

O Conceito de indução eletromagnética primeiro descrito por Faraday em 1831. Uma bobina de fio gera um intenso campo magnético alternado, que consequentemente induz uma corrente elétrica secundária na base tecido onde interage com os neurônios (Peres \& Gomes, 2020). A lei de Faraday-Neumann-Lenz, ou lei da indução de Faraday, é uma das equações básicas do eletromagnetismo. Expressões matemáticas descrevem e predizem como o campo magnético interage com o campo elétrico e, como resultado, a interação dessas forças produz outra força chamada força eletromotriz: a indução eletromagnética.

O PEMF utiliza campos magnéticos alternados, baseado na lei da indução eletromagnética, promove correntes elétricas que despolarizam o tecido neuromuscular causando contrações supramáximas (Kinney \& Lozanova, 2019; Kent \& Kinney, 2021). Os neurônios motores são ativados devido ao seu grande diâmetro e, portanto, menor resistência em comparação com outros tipos de neurônios. Uma vez que os nociceptores não são ativados, a aplicação de estimulação magnética não é dolorosa (Tyler, 2017; Weiss \& Bernardy, 2019;). 
O PEMF gera impulsos que são independentes da função cerebral, e com uma frequência tão rápida que não permite a fase de relaxamento muscular, caracterizando as contrações tetânicas. Para simular condições de carga de trabalho ao músculo, o equipamento modula a emissão de PEMF em modos submáximos, de $1 \mathrm{~Hz}$ a $10 \mathrm{~Hz}$ e modo supramáximo, de $10 \mathrm{~Hz}$ a $100 \mathrm{~Hz}$, com tempo ON em segundos também parametrizável (0 a 60s).

Sob condições normais, a maior quantidade de tensão que poderia ser desenvolvido e realizada fisiologicamente é chamada contração voluntária máxima (CVM) (Weiss \& Bernardy, 2019). Normalmente, dura apenas uma fração de segundo. Contrações com tensão superiores a (CVM) são definidos como supra máximos. A PEMF tem a capacidade de gerar contrações supra-máximas sustentadas por vários segundos, o que aumenta significativamente o estresse / carga de trabalho, se que haja adaptação muscular (Giesse, 2021). Na contração supra-máxima as proteínas são degradadas, porém os aminoácidos são reaproveitados no processo de síntese as custas de gasto energético intenso.

Vários estudos mostraram que os humanos são incapazes de ativar totalmente os músculos voluntariamente como a força dos músculos a contração é limitada pelas taxas de disparo e condutividade de vias neurais (Gabriel et al, 2006; Dowling et al, 1994; Gandevia, 2001;). Muitos profissionais acreditam que o acúmulo de ácido lático é responsável pelo processo de fadiga muscular. No entanto, o verdadeiro vilão é a alteração de pH na região da junção neuromuscular que pode, inclusive, ser potencializada pela quebra continua de ATP em ADP e liberação de um P+, que intensifica a acidificação da placa mioneural. Esta acidificação induz a um bloqueio de transmissão nervosa, culminando em fadiga muscular. Ao contrário do que se diz, o PEMF independe de ativação neural, induzindo um estímulo direto na fibra muscular e realizando um "by-pass" do processo de bloqueio neuromuscular da fadiga fisiológica.

A Figura 1 ilustra o ciclo de contrações musculares induzidos em um protocolo utilizando a tecnologia PEMF.

Figura 1: Esquema ilustrativo das contrações musculares induzidas pelo PEMF.

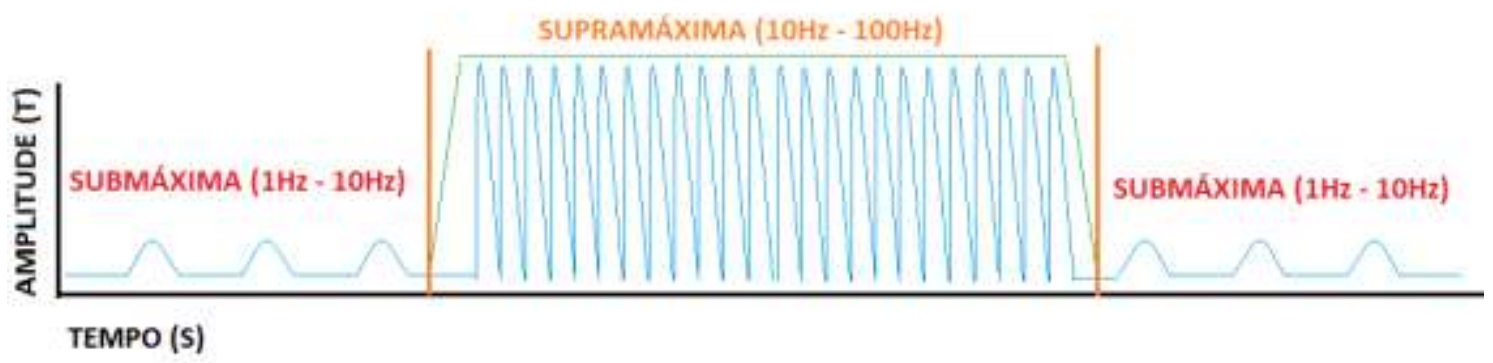

Fonte: Autores.

Contrações musculares intensas exigem uma quantidade considerável de energia que é fornecida na forma de ácidos graxos livres a partir do tecido adiposo via lipólise, o que além do consumo de FFA por ativação de beta oxidação também favorece a redução de triglicerídeos geral (Kniight \& Kamen, 2001; Goldberg, 2021;). A PEMF aumenta atividade catalítica LDH em 35\% e CK em 227,8 \% ao longo de 8 horas. (Weiss \& Bernardy, 2019).

\section{PEMF e Hipertrofia Muscular}

Segundo Duncan e Dinev (2020), em um estudo in vivo realizado em modelo experimental porcino, o PEMF foi capaz de induzir alterações musculares hipertróficas após 2 semanas de tratamento. Os autores relatam um aumento da densidade da massa muscular de 20,56\%. No mesmo estudo, foi observado um aumento na densidade da fibra muscular (hiperplasia) de 8,0\%. O tamanho médio da fibra muscular individual aumentou 12,15\%, 2 semanas após o tratamento, enquanto o grupo controle não apresentou mudanças significativas na densidade da fibra ou hiperplasia. Os autores sugerem 
que o PEMF pode ser usado para indução não invasiva do crescimento muscular.

O mecanismo exato de contração muscular pelo campo eletromagnético ainda permanece desconhecido. A hipótese clássica é a de despolarização dos neurônios motores periféricos, com liberação de acetilcolina na placa mioneural. A acetilcolina se liga a receptores na membrana das células musculares esqueléticas (Receptores-canais de sódio), alterando a voltagem da membrana e induzindo a abertura dos canais de cálcio voltagem-dependentes, resultando em um influxo maciço de cálcio e induzindo a contração muscular. Entretanto, existem evidências de que o PEMF é capaz de estimular ao menos dois mecanismos moleculares relacionados a expressão de Canais de Cálcio Voltagem dependentes e aumento das concentrações de cálcio intra-celulares em tecido ósseo (Petechia et al, 2015). No entanto, o efeito observado em células ósseas pode estar se repetindo em tecido muscular, o que explicaria uma maior efetividade do PEMF em relação às correntes elétricas. Isto porque, o efeito de contração muscular, na verdade, pode ser desencadeado por uma alteração direta de voltagem da membrana da célula muscular esquelética, levando a abertura dos canais de cálcio voltagem-dependentes e consequente contração muscular, porém sem depender da despolarização neuronal, ou seja, um efeito direto sobre o músculo. Obviamente, essa hipótese reduziria bastante o fator de fadiga muscular decorrente da inibição de transmissão nervosa na placa mioneural ocasionada pela acidificação nesta região. Petechia et al (2015) ainda demonstram o aumento da expressão de canais de cálcio voltagem dependentes em células em cultura. Se o efeito for reprodutível em musculatura esquelética, abre-se uma avenida de possibilidades para o ganho efetivo de geração de força e eficiência muscular, tanto na área esportiva quanto estética.

\section{PEMF e Diástase Abdominal Pós-Parto}

Durante a gravidez, os músculos abdominais são esticados e separados extensivamente para acomodar o feto em crescimento.4 No entanto, os músculos abdominais frequentemente não retornar completamente à sua posição original e podem permanecer separados após o parto. Em casos severos, a separação dos músculos pode exceder 2,7 Cm, caracterizando uma condição de diástase abdominal. Jacob e Rank (2020) em um estudo piloto com 10 pacientes utilizou o protocolo de PEMF duas vezes por semana durante 30 minutos por 2 semanas. As pacientes foram acompanhadas 1, 3 e 6 meses após o término do tratamento com PEMF.

Os resultados obtidos a partir de imagens de ressonância magnética mostraram uma redução média de gordura de $17 \%$ em um mês e $20 \%$ após 3 meses. Os autores também relataram um efeito de aumento médio da espessura do músculo de 20,5 \% após 01 mês e 21,3\% após 3 meses. Especialmente, no que diz respeito a distância entre os músculos retos abdominais foi observada uma redução de

16,7\% em 01 mês e 22,7\% após 3 meses. Após seis meses, os autores reportam que 9 pacientes retornaram para avaliação, e apresentaram, em média, uma redução de gordura de 17,6\%, aumento de 21,7\% no músculo e redução de

23,2\% da distância entre os músculos abdominais. O peso dos pacientes não mudou significativamente. O referido estudo não apresentou grupo controle, mas os resultados inicias sugerem um efeito benéfico na redução de gordura e no fortalecimento muscular.

\section{PEMF e Glúteos}

No Brasil, uma das maiores demandas de estética corporal é aparência da região de glúteos e coxas. Na última década, especialmente, o aumento de nádegas vem crescendo significativamente ao longo da última década (McRae \& Boris, 2013). Segundo os dados reportados pela Sociedade Americana de Cirurgiões Plásticos, para o ano de 2018 foram realizados mais de 29.000 procedimentos estéticos para glúteos, incluindo a colocação de implante de silicone; retalhos locais ou rearranjo de tecidos, preenchimento com substâncias como géis de ácido hialurônico, polimetilmetacrilato ou poliacrilamida e ainda, 
enxerto autólogo de gordura (Plastic Surgery Statistics, 2020; Chacur et al, 2019;). Entretanto, a incidência de complicações e efeitos adversos para estes procedimentos é relativamente alta, incluindo fibroses, necrose tecidual, seroma, dor e hipersensibilidade e infecções.

Tendo em vista os riscos inerentes aos procedimentos invasivos, as tecnologias não invasivas e de baixo risco como o PEMF, representam uma importante alternativa para o ganho de massa muscular na região glútea. Um estudo recente utilizando imageamento por ressonância magnética nuclear demonstrou na análise volumétrica, um aumento significativo no tamanho dos músculos do glúteo de 10,81 $\pm 1,60 \%$, após 01 mês de tratamento, e no acompanhamento, após 3 meses, um aumento de 13,23 \pm 0,91\%. Os autores observaram um efeito hipertrófico mais profundo na parte superior do glúteo. Esse efeito resultou em uma elevação visível das nádegas, também comprovado por análise de imagens fotográficas (Palm, 2021).

\section{PEMF e Incontinência Urinária}

A Incontinência urinária (IU) é normalmente definida como a perda involuntária de urina. Trata-se de um problema crônico que afeta negativamente e de forma significativa a qualidade de vida (Abrams et al, 2002). A incontinência urinária é classificada como a) de estresse (IUE); b) de urgência (IUU); c) Mista (IUM).

A prevalência da incontinência urinária pode variar entre 25 e 45\%, mas podendo chegar a $69 \%$ em algumas populações, e os sintomas parecem se agravar com o avanço da idade, índice de massa corporal e alguns outros fatores (Norwegian Epicont study, 2000; Melville et al, 2005; Swithinbank et al, 1999; Khullar et al, 2014; Saadia, 2015;). Em geral, o mecanismo de incontinência urinária está frequentemente associado à falha do aparato muscular do assoalho pélvico.

Recentemente, Samuels et al (2019) realizaram um estudo com 75 pacientes portadores de incontinência urinária e fortalecimento muscular utilizando a tecnologia PEMF. De todos os pacientes (75), 61 mostraram melhora significativa de 49,93\% dos sintomas após 6 semanas de tratamento e 64,4\% após 3 meses. Os autores concluíram que a tecnologia de PEMF foi capaz de induzir uma melhora significativa nos sintomas de incontinência urinária, especialmente mista. No acompanhamento de 06 meses após o tratamento foi observada uma melhora da qualidade de vida dos pacientes, com relação ao problema.

Neste mesmo tema, Elena et al (2020) investigou a eficiência da tecnologia de PEMF comparada à estimulação elétrica clássica. O estudo foi realizado com 95 mulheres em período pós-parto que apresentavam queixa de incontinência urinária. As pacientes sintomáticas receberam tratamento com PEMF ou eletroestimulação em 10 sessões com a periodicidade de 2 a 3 vezes por semana (PEMF - 28 minutos/sessão) ou em dias alternados (eletroestimulação). Neste estudo, 50 pacientes foram tratados com PEMF, 25 foram tratados com eletroestimulação e 20 foram considerados como grupo controle saudável para fins de comparação. Os autores reportam que a tecnologia de PEMF foi capaz de melhorar significativamente os índices biométricos de integridade do assoalho pélvico e os sintomas de incontinência urinária, e que tais resultados se devem ao fortalecimento da musculatura do assoalho pélvico. Interessantemente, os pacientes também reportaram menor desconforto com a utilização do PEMF, quando comparados a corrente elétrica.

\section{Conclusões}

A tecnologia de Campo eletromagnético pulsado (PEMF) é uma tecnologia inovadora e que tem apresentado importantes resultados no ganho de força e massa muscular. Suas aplicações são diversas, variando desde aspectos estéticos como a tonificação de músculos glúteos, ganho de força e massa muscular em atletas e melhora de problemas como a incontinência urinária, devido ao fortalecimento da musculatura do assoalho pélvico.

A hipótese que levantamos de que o campo eletromagnético é capaz de realizar um by-pass neuronal, produzindo uma 
contração muscular direta por abertura de canais de cálcio voltagem-dependentes, pode ampliar e beneficiar um grande número de pacientes e patologias. Estudos adicionais, randomizados, cegos e controlados se fazem necessários para estabelecermos e demonstrarmos a grande variedade potencial de aplicações desta notável tecnologia.

Além disso, sugerimos que essa tecnologia pode ser estudada em pacientes hospitalizadas imobilizados, acamados que normalmente apresentam rápidas perdas funcionais musculares, assim como na prevenção de trombose venosa profunda. Ainda, podemos sugerir estudos com sequelas musculares importantes como pós-covid 19 e na manutenção de massa muscular em pacientes neurológicos. São necessários estudos clínicos randomizados, com metodologia cega e controlada ou comparada com outras tecnologias existentes, com o objetivo de comprovar as vantagens desta nova tecnologia sobre aquelas existentes.

\section{Referências}

Abrams, P., Cardozo, L., Fall, M., Griffiths, D., Rosier, P., Ulmsten, U., van Kerrebroeck, P., Victor, A., \& Wein, A. (2002) Standardisation Sub-committee of the International Continence Society. The standardisation of terminology of lower urinary tract function: report from the Standardisation Sub-committee of the International Continence Society. Neurourol Urodyn. 21(2):167-78. 10.1002/nau.10052.

A

lizadeh Z, Halabchi F, Mazaheri R, Abolhasani M, \& Tabesh M. (2016) Review of the Mechanisms and Effects of Noninvasive Body Contouring Devices on Cellulite and Subcutaneous Fat. Int J Endocrinol Metab. 14(4):e36727. 10.5812/ijem.36727.

American Society of Plastic Surgeons. Plastic Surgery Statistics Report. Plastic Surgery. (2020) https://www.plasticsurgery.org/news/plastic-surgery-statistics.

Chacur, R., Sampaio Menezes, H., Maria Bordin da Silva Chacur, N., Dias Alves, D., Cadore Mafaldo, R., Dias Gomes, L., \& Dos Santos Barreto, G. (2019) Gluteal Augmentation with Polymethyl Methacrylate: A 10-year Cohort Study. Plast Reconstr Surg Glob Open. 31;7(5):e2193. 10.1097/GOX.0000000000002193.

Dowling, J.J., Konert, E., Ljucovic, P., \& Andrews, D.M. (1994) Are humans able to voluntarily elicit Maximum muscle force? Neurosci Lett. 179(1-2):25-28.

Duncan, D., \& Dinev, I. (2020) Noninvasive Induction of Muscle Fiber Hypertrophy and Hyperplasia: Effects of High-Intensity Focused Electromagnetic Field Evaluated in an In-Vivo Porcine Model: A Pilot Study. Aesthet Surg J. 40(5):568-574. 10.1093/asj/sjz244.

Elena, S., Dragana, Z., Ramina, S., Evgeniia, A., \& Orazov, M. (2020) Electromyographic Evaluation of the Pelvic Muscles Activity After High-Intensity Focused Electromagnetic Procedure and Electrical Stimulation in Women With Pelvic Floor Dysfunction. Sex Med. 8(2):282-289. 10.1016/j.esxm.2020.01.004. Epub 2020 Mar 4.

Gabriel, D.A., Kamen, G., \& Frost, G. (2006) Neural adaptations to resistive exercise: mechanisms and recommendations for training practices. Sports Med. 36(2):133-149.

Gandevia, S.C. (2001) Spinal and supraspinal factors in human muscle fatigue. Physiol Rev. 81(4):1725-1789.

Gentil, P., Costa, D., \& Arruda, A. (2017). CrossfitÂ®: uma análise crítica e fundamentada de custo-benefício. RBPFEX - Revista Brasileira De Prescrição E Fisiologia Do Exercício, 11(64), 138-139.

Giesse S., (2021) A German Prospective Study of the Safety and Efficacy of a Non-Invasive, Highintensity, Electromagnetic Abdomen and Buttock Contouring Device. 2021. J Clin Aesthet Dermatol. 14(1):30-33.

Goldberg, D. J. (2021) Deletion of adipocytes induced by a novel device simultaneously delivering synchronized radiofrequency and hifem: Human histological study. J Cosmet Dermatol. 20(4):1104-1109. 10.1111/jocd.13970.

Halaas, Y. \& Bernardy, J. (2020). Mechanism of nonthermal induction of apoptosis by highintensity focused electromagnetic procedure: Biochemical investigation in a porcine model. J Cosmet Dermatol.19:605-611.

Hopia H, Latvala E, \& Liimatainen L. (2016) Reviewing the methodology of an integrative review. Scand J Caring Sci. 30(4):662-669. 10.1111/scs.12327.

Jacob, C. I., \& Rank, B. (2020) Abdominal Remodeling in Postpartum Women by Using a High-intensity Focused Electromagnetic (HIFEM) Procedure: An Investigational Magnetic Resonance Imaging (MRI) Pilot Study. J Clin Aesthet Dermatol. 13(9 Suppl 1): S16-S20.

Kent, D. E. \&. Kinney, B. M. (2021). The effect of high-intensity focused electromagnetic procedure on visceral adipose tissue: Retrospective assessment of computed tomography scans. J Cosmet Dermatol. 20:757-762.

Khullar, V., Sexton, C. C., Thompson, C. L., Milsom, I., Bitoun, C. E., \& Coyne, K. S. (2014) The relationship between BMI and urinary incontinence subgroups: Results from EpiLUTS. Neurourol Urodyn 33(4):392-399. https://doi.org/10.1002/nau.22428

Kinney, B. M., \& Lozanova, P. (2019). High Intensity Focused Electromagnetic Therapy Evaluated by Magnetic Resonance Imaging: Safety and Efficacy Study of a Dual, B. M., Tissue Effect Based Non-Invasive Abdominal Body Shaping. Lasers in Surgery and Medicine 51:40-46.

Knight, C.A., \& Kamen, G. (2001) Adaptations in muscular activation of the knee extensor muscles with strength training in young and older adults. $J$ Electromyogr Kinesiol. 11(6):405-412. 
Research, Society and Development, v. 10, n. 14, e06101421724, 2021

(CC BY 4.0) | ISSN 2525-3409 | DOI: http://dx.doi.org/10.33448/rsd-v10i14.21724

McRae, E., \& Boris, J. (2013) Independent evaluation of low-level laser therapy at $635 \mathrm{~nm}$ for non-invasive body contouring of the waist, hips, and thighs. Lasers Surg Med. 45(1):1-7.

Melville, J. L., Katon, W., Delaney, K., \& Newton, K. (2005) Urinary incontinence in US Women: A population-based study. Arch Intern Med 165(5):537542. https://doi.org/10.1001/archinte.165.5.537

Norwegian EPINCONT study. Epidemiology of Incontinence in the County of Nord-Trøndelag. (2000) A community-based epidemiological survey of female urinary incontinence: The Norwegian EPINCONT study. Epidemiology of Incontinence in the County of Nord-Trøndelag. J Clin Epidemiol 53(11):11501157.

Palm, M. (2021). Magnetic Resonance Imaging Evaluation of Changes in Gluteal Muscles After Treatments With the High-Intensity Focused Electromagnetic Procedure. Dermatol Surg. 47(3):386-391. 10.1097/DSS.0000000000002764.

Peres, R. A., \& Gomes, L. C. (2020) Análise de alguns equívocos e distorções presentes em sites da web e vídeos do youtube sobre a lei da indução eletromagnética de Michael Faraday. \#Tear: Revista de Educação, Ciência e Tecnologia, Canoas, 9(1), 10.35819/tear.v9.n1.a3958.

Petecchia, L., Sbrana, F., Utzeri, R., Vercellino, M., Usai, C., Visai, L., Vassalli, M., \& Gavazzo, P. (2015) Electro-magnetic field promotes osteogenic differentiation of BM-hMSCs through a selective action on Ca(2+)-related mechanisms. Sci Rep. 14;5: 13856. 10.1038/srep13856.

Plastic Surgery Statistics. American Society of Plastic Surgeons. (2020). https://www.plasticsurgery.org/news/plastic-surgerystatistics.

Radwan, H., Hasan, H.A., Ismat, H., Hakim, H., Khalid, H., Al-Fityani, L., Mohammed, R., \& Ayman, A. (2019) Body Mass Index Perception, Body Image Dissatisfaction and Their Relations with Weight-Related Behaviors among University Students. Int J Environ Res Public Health. 1;16(9):1541. 10.3390/ijerph16091541.

Saadia, Z. (2015) Effect of age, educational status, parity and BMI on development of urinary incontinence-A cross sectional study in Saudi Population. Mater Sociomed 27(4):251-254. https://doi.org/10.5455/msm.2015.27.251-254

Samuels, J. B., Pezzella, A., Berenholz, J., \& Alinsod, R. (2019) Safety and Efficacy of a Non-Invasive High-Intensity Focused Electromagnetic Field (HIFEM) Device for Treatment of Urinary Incontinence and Enhancement of Quality of Life. Lasers Surg Med. 51(9):760-766. 10.1002/1sm.23106.

Swithinbank, L.V., Donovan, J.L., du Heaume, J.C., Rogers, C.A., James, M. C., Yang, Q., \& Abrams, P. (1999) Urinary symptoms and incontinence in women: relationships between occurrence, age, and perceived impact. Br J Gen Pract. 49(448):897-900.

The American Society for Aesthetic Plastic Surgery. (2018) Procedural Statistics. The Aesthetic Society. https://www. surgery.org/sites/default/ᄀ les/ASAPSStats2017.pdf.

Tyler, D. J. (2017) Peripheral nerve stimulation. In: Series on bioengineering and biomedical engineering. 8. (2a ed.), World Scientific. 300-347.

Weiss, R.A., \& Bernardy, J. (2019) Induction of fat apoptosis by a non-thermal device: Mechanism of action of non-invasive high-intensity electromagnetic technology in a porcine model. Lasers Surg Med. 51(1):47-53. 10.1002/lsm.23039;

Whittemore R, \& Knafl K. The integrative review: updated methodology. (2005) J Adv Nurs. 52(5):546-53. 10.1111/j.1365-2648.2005.03621.x. 